\title{
DÜBLIN
}

Technological University Dublin ARROW@TU Dublin

\section{Inner Surface Biofilm Inactivation by Atmospheric Pressure Helium Porous Plasma Jet}

\author{
Peng Lu \\ Technological University Dublin \\ Dana Ziuzina \\ Technological University Dublin, dana.ziuzina@tudublin.ie \\ Patrick Cullen \\ Technological University Dublin, pj.cullen@tudublin.ie
}

See next page for additional authors

Follow this and additional works at: https://arrow.tudublin.ie/schfsehart

\section{Recommended Citation}

Bourke, P. (2018) Inner Surface Biofilm Inactivation by Atmospheric Pressure Helium Porous Plasma Jet, Plasma Process Polym.2018;15:e1800055. DOI:10.1002/ppap.201800055

This Article is brought to you for free and open access by the School of Food Science and Environmental Health at ARROW@TU Dublin. It has been accepted for inclusion in Articles by an authorized administrator of ARROW@TU Dublin. For more information, please contact arrow.admin@tudublin.ie, aisling.coyne@tudublin.ie, gerard.connolly@tudublin.ie.

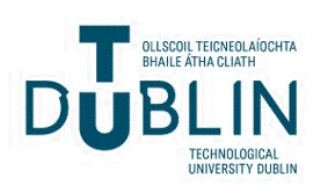




\section{Authors}

Peng Lu, Dana Ziuzina, Patrick Cullen, and Paula Bourke 
Article type: Full Paper

\title{
Title: Inner Surface Biofilm Inactivation by Atmospheric Pressure Helium Porous Plasma Jet
}

Peng Lu ${ }^{1 \mathrm{a}}$, Dana Ziuzina ${ }^{1 \mathrm{a}}$, P J Cullen ${ }^{1,2}$, Paula Bourke ${ }^{1 *}$

1. Plasma Research Group, School of Food Science and Environmental Health, Dublin Institute of Technology, Dublin 1, Ireland

2. Department of Chemical and Environmental Engineering, University of Nottingham, UK.

a. Contributed equally to this work

*E-mail: (paula.bourke@ dit.ie)

\begin{abstract}
We present a helium porous plasma jet based on gas diffuser, designed with the aim of decontaminating inner surfaces of contaminated structures e.g pipes. The porous plasma jet operates with three discharge modes in ambient air and inside a vessel. Its decontamination capacity is demonstrated by evaluating the inactivation efficacy of biofilm form of Pseudomonas aeruginosa, adherent to inner surface of a glass vial. Plasma treatments for 5 mins with filament mode and double region helium discharge mode reduced bacterial numbers by 2.4 and $2.5 \log 10 \mathrm{CFU} / \mathrm{ml}$. Plasma treatment with double region air-like discharge mode was the most effective, reducing bacterial cells by $4.5 \mathrm{Log} 10 \mathrm{CFU} / \mathrm{ml}$, which demonstrates porous plasma jet could provide an efficient approach for inner surface decontamination.
\end{abstract}

\section{$1 \quad$ Introduction}

Cold (or non-thermal equilibrium) atmospheric plasma (CAP) have been extensively applied in biological, medical and environmental fields ${ }^{[1-3]}$. Among the widespread applications of CAPs, possibly the most-investigated and best-known biological application is the 
inactivation of bacterial cells for applications in sterilization in fields, such as medical devices ${ }^{[4]}$, living tissue (skin, wounds, dental root) ${ }^{[5,6]}$, food and agriculture ${ }^{[7]}$ and cancer cell treatments ${ }^{[8,9]}$. Both direct discharge treatment and flowing afterglow plasma treatment have demonstrated antimicrobial effects for diverse bacterial strains in various dry and wet target materials. Biofilms represent a challenging resevoir of microbial contamination, which build up and establish in environmental niches in processing environments and indwelling devices. These challenges are often present on the internal surfaces of tubes, pipes, devices etc. For a practical CAP application when the treatment target is attached on the inner surface of a chamber, or device tubing or pipeworks, the generated plasma is required to extend into the interior of tubing and contact with the surface. Currently, the plasma source for inner-surface decontamination is of great technological interest. Kitazaki and coworkers reported a method using vacuum chamber to produce low pressure plasma inside a narrow pipe ${ }^{[10,11]}$. Polak and coworkers proposed a method in which two wire electrodes were twined around the treated tube to produce CAP inside it ${ }^{[12]}$. A typical CAP source configuration is the plasma jet which can launch the stable plasma species to a separate environment ${ }^{[3,13]}$. When the desired application requires an all-side treatment, the multi-dimensional combination or rotation of plasma jets is necessary to irradiate $3 \mathrm{D}$ surfaces.

In real conditions, bacterial cells persist in the form of biofilms rather than planktonic form. Biofilm represents a major form of surface biological contamination, where cells are embedded in a matrix of extracellular polymeric substances forming a complex defensive mechanism making bacteria within them resistant to antimicrobial treatments ${ }^{[14,15]}$. Previous studies investigated the efficacy of CAP against bacterial biofilms produced by diverse monoculture bacterial strains relevant to food and pharmaceutical industries ${ }^{[16-20]}$. The aim of this work was to evaluate the feasibility of a porous helium plasma jet based on a cylindrical gas diffuser for inactivation of bacterial biofilms as contaminants of the inner surface of a glass cylinder. Pseudomonas aeruginosa (P. aeruginosa), a Gram-negative abundant 
microorganism in the natural environment and a common opportunistic nosocomial pathogen, was selected as the target biofilm-forming bacteria. The effect of different discharge operational modes on inactivation within $P$. aeruginosa biofilm was investigated to identify the optimal approach.

\section{Experimental Section}

\subsection{Porous plasma jet}

The porous plasma jet is based on a cylindrical metal gas diffusor (WAT025531, WATERS). The diameter and length of the diffusor are $12 \mathrm{~mm}$ and $20 \mathrm{~mm}$, respectively, and its pore size is about $10 \mu \mathrm{m}$. The diffusor is attached to one end of a quartz tube, opposite to the gas inlet feed, as shown in Figure 1. The inner and outer diameter of the quartz tube are $3 \mathrm{~mm}$ and 6 $\mathrm{mm}$, respectively. The single electrode configuration was adopted in this porous plasma jet, in which an aluminum foil wrapped on the quartz tube served as the high voltage electrode. The distance between the high voltage electrode and the inlet of gas diffuser is $5 \mathrm{~cm}$. The gas diffusor provides a porous media for the gas flow and also enhance the local electric field thereby favoring the plasma jetting. Helium gas (CP grade $99.999 \%$, BOC gas) was used as the working gas. The gas flow rate was regulated by a mass flow controller (KOFLOC DF300C). A HV half bridge resonant inverter circuit (PVM500, INFORMATION

UNLIMITED) with a set-up transformer delivered a sinusoidal AC high voltage to drive the plasma discharge. Applied voltage and discharge current were monitored by a HV probe (Tektronix P6015A) and a wideband current transformer (0.5-1.0W, STANGENES INDUSTRIES), respectively. InfiniVision DSO-2014A oscilloscope (Agilent Technologies, 100-MHz bandwidth and 2-G Samples/s sampling rate) was used to record voltage and current waveforms. The optical emission spectrum from the discharge plasma was measured by a miniature CCD spectrometer (Exemplar LS, BWTEK). The light from the plasma 
emission was focused by an optical fiber coupled lens and guided to the spectrometer via an optical fiber.

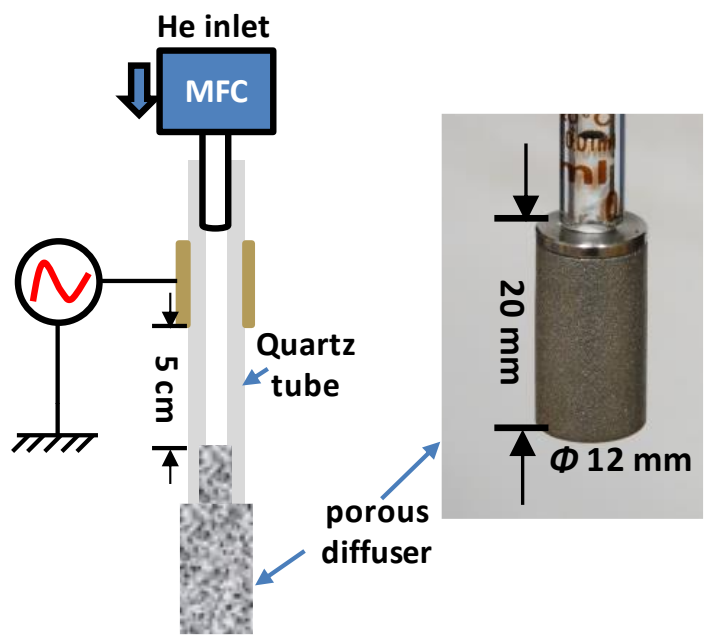

Figure 1. Schematic of the helium porous plasma jet

\subsection{Biofilm culture and analysis}

P. aeruginosa (ATCC 27853) biofilms were obtained in universal glass bottles ( $25 \mathrm{ml}$ capacity with wide neck) by inoculating either 3 (for plasma mode 1 and 2) or $6 \mathrm{ml}$ (for plasma mode 3) of sterile TSB with 100 or $200 \mu 1$ of overnight culture and incubating the culture in rotary shaking incubator for $24 \mathrm{~h}$ at $37^{\circ} \mathrm{C}$ and $200 \mathrm{rpm}$. Prior to plasma treatment, the TSB containing suspended bacterial cells was removed and bottles were washed with sterile PBS, leaving only surface attached bacterial biofilms for further investigation. $P$. aeruginosa biofilms on the inner wall of the glass vials were easily visible as shown in Figure 2.

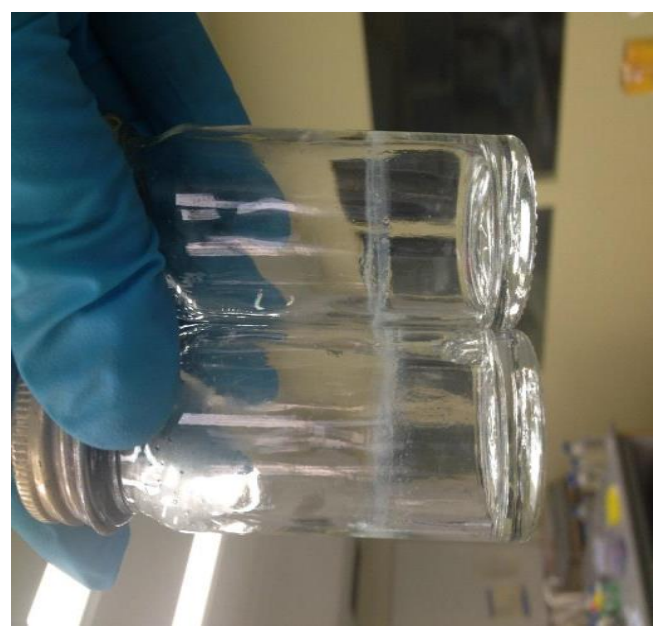


Figure 2 P. aeruginosa biofilms cultured on the inner surface of glass vials

For treatment, the jet was introduced inside the vial and plasma was ignited. P. aeruginosa 24 $\mathrm{h}$ biofilms were treated by plasma discharge for 5 min with different discharge modes (described in section 3.1) individually. To analyse surviving population of cells following treatment, biofilms were disrupted by adding sterile maximum recovery diluent (MRD, ScharlauChemie, Spain) and sterile glass beads into the bottles, which were then vortexed for 1 min. Surviving populations of $P$. aeruginos $a$ were estimated by using drop plate technique, where aliquots of appropriate dilutions $(10 \mu \mathrm{l})$ were surface plated on tryptic soy agar (TSA, ScharlauChemie, Spain) and incubated at $37^{\circ} \mathrm{C}$ for $24 \mathrm{~h}$. Experiments were repeated at least 5 times. Results are presented as surviving bacterial population in $\log _{10} \mathrm{CFU} / \mathrm{ml}$ (limit of detection 2.0 $\log _{10} \mathrm{CFU} / \mathrm{ml}$ ). In addition, XTT assay (2,3-bis (2-methoxy-4-nitro-5sulfophenyl) [phenyl-amino)car-bonyl]-2H-tetrazolium hydroxide (XTT), 1 mg/ml, SigmaAldrich, Ireland) was performed as described previously ${ }^{[21]}$ to examine cells metabolic state. To study the independent effect of temperature, bacterial biofilms were exposed to $50^{\circ} \mathrm{C}$ in water bath for $15 \mathrm{~min}$ and the number of surviving cells was estimated by colony count assay. The effect of plasma treatments on bacterial cell morphology was also analysed by scanning electron microscopy (SEM). For this, $P$. aeruginosa biofilms were formed on membrane filters, which were introduced inside the bottles prior to media inoculation. After $24 \mathrm{~h}$ incubation biofilms were either exposed to 5 min of plasma treatment or left untreated (controls). After treatment the filters were aseptically removed from the bottles and the area containing biofilms $(\sim 10 \mathrm{x} 10 \mathrm{~mm})$ was excised for further preparations. The biofilms were prepared for SEM according to the procedure described by Los et al ${ }^{[22]}$. In order to prevent surface charging by the electron beam, the samples were sputter-coated with gold particles using Emitech K575X Sputter Coating Unit resulting in a coating of $10 \mathrm{~nm}$ after $30 \mathrm{~s}$. The samples were examined visually using a FEI Quanta 3D FEG Dual Beam SEM (FEI Ltd, Hillsboro, USA) at $5 \mathrm{kV}$. 


\section{$3 \quad$ Results and Discussion}

\subsection{Characteristics of porous plasma jet}

By varying the applied voltage amplitude and gas flow rate the porous plasma jet can be operated with different discharge modes in the ambient air. Figure 3 shows a series of typical plasma images, in which the amplitude of applied voltage was gradually raised with the fixed frequency at $28 \mathrm{kHz}$ and gas flow rate $10.5 \mathrm{~L} / \mathrm{min}$. As a reference, Figure 3 includes a conventional plasma jet without the gas diffusor in the case of $6.7 \mathrm{~L} / \mathrm{min}$ gas flow rate. Figure 3 shows that the morphology of porous plasma jet discharge in the ambient air is generally composed of a ring-like plasma region on the diffusor surface and multiple ray-like plasma jets spreading into the ambient air. In the case of low applied voltage $\left(V_{P} 3.8 \mathrm{kV}\right)$ as shown in Figure 3(a), a plasma ring which is composed of a series of localized plasma spots from each tiny hole of the gas diffusor arises from the diffusor surface. In tandem with increasing the voltage amplitude, the width of the plasma ring (indicated by $L$ in Figure 3 (i)) increases along the axial direction. Meanwhile, multiple regular straight plasma jets spread out of the plasma ring into the ambient air, which appear like regular rays of light as shown in Figure 3(b)-(c). Additionally, the length of each plasma jet increases slightly in accordance with increasing applied voltage. In the case of higher voltage, Figure 3(e) and (f) show the transition of the regular straight line-like plasma plumes into the irregular swing-like mode. With a further voltage increase, the location of the bright spot on the diffusor surface disperses and the bright spots becomes larger and brighter; in addition, the ejected plasma jets swing upwards. These plasma images suggest that the plasma patterns can be categorized and controlled into three types, namely 'no plasma plume' mode, regular 'ray-like plume' mode and 'swing-like plume' mode. To visualize the helium gas flow into ambiemt air through the gas diffusor, a Ztype schlieren imaging system was set up. Figure 4 shows schlieren images of helium gas flow. The gas diffusor is placed vertically downwards. The helium gas flows upwards after coming out of the diffusor. It looks like that helium gas wraps the diffusor. Increasing the gas 
flow rate increases the radial distribution of helium gas. The pattern of helium gas flow into the ambient air through the gas diffusor looks different from the laminar or turbulent flow of the conventional plasma jet through a dielectric tubing which has been reported widely. With the plasma on (Figure 4(g) and (h)), it is hard to distinguish flow pattern of each jet from the diffusor, by comparison with the ray-like plasma jets shown in Figure 3. However, the gas flow pattern is changed and becomes irregular after plasma ignition. This may be partly due to helium temperature increase caused by the heating of gas diffusor.

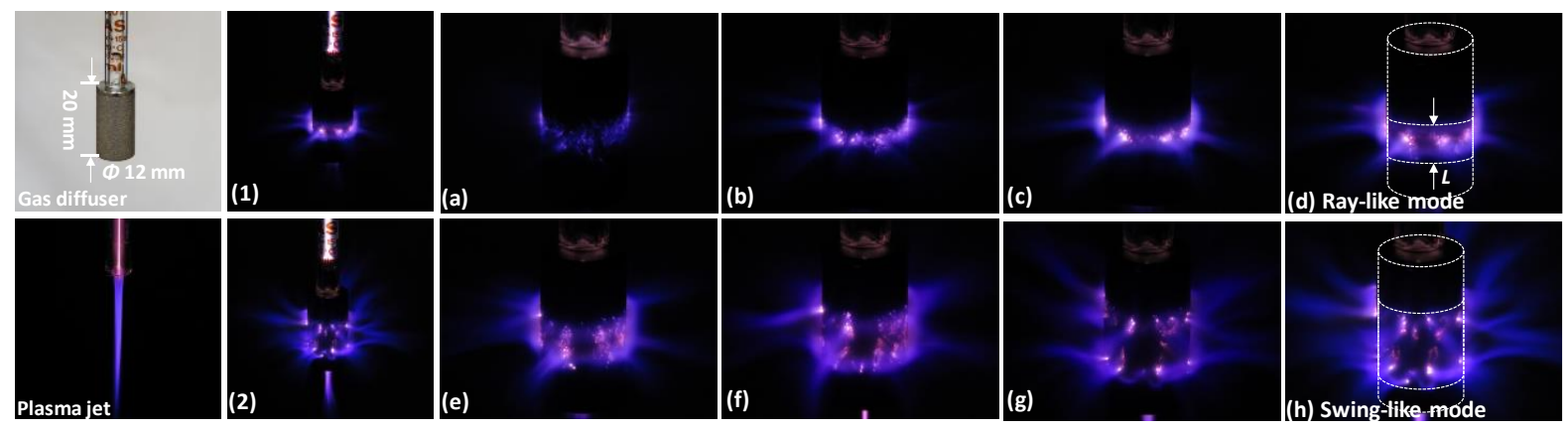

Figure 3. Photograph of porous gas diffusor, porous plasma jet operated at $10.5 \mathrm{~L} / \mathrm{min}$ with the applied voltage $V_{P}$ of (a) $3.8 \mathrm{kV}$, (b) $5.8 \mathrm{kV}$, (c) $6 \mathrm{kV}$, (d) $6.2 \mathrm{kV}$ (e) $6.8 \mathrm{kV}$, (f) $7.2 \mathrm{kV}$, (g) 8 $\mathrm{kV}$, (h) $8.5 \mathrm{kV}$, and a conventional helium plasma jet at $6.7 \mathrm{~L} / \mathrm{min}$ and $8 \mathrm{kV}$; (1) and (2) shows the far-away images corresponding (d) and (h). Taken by Canon PowerShot SX60 HS, Exposure time 1/3s, ISO 200
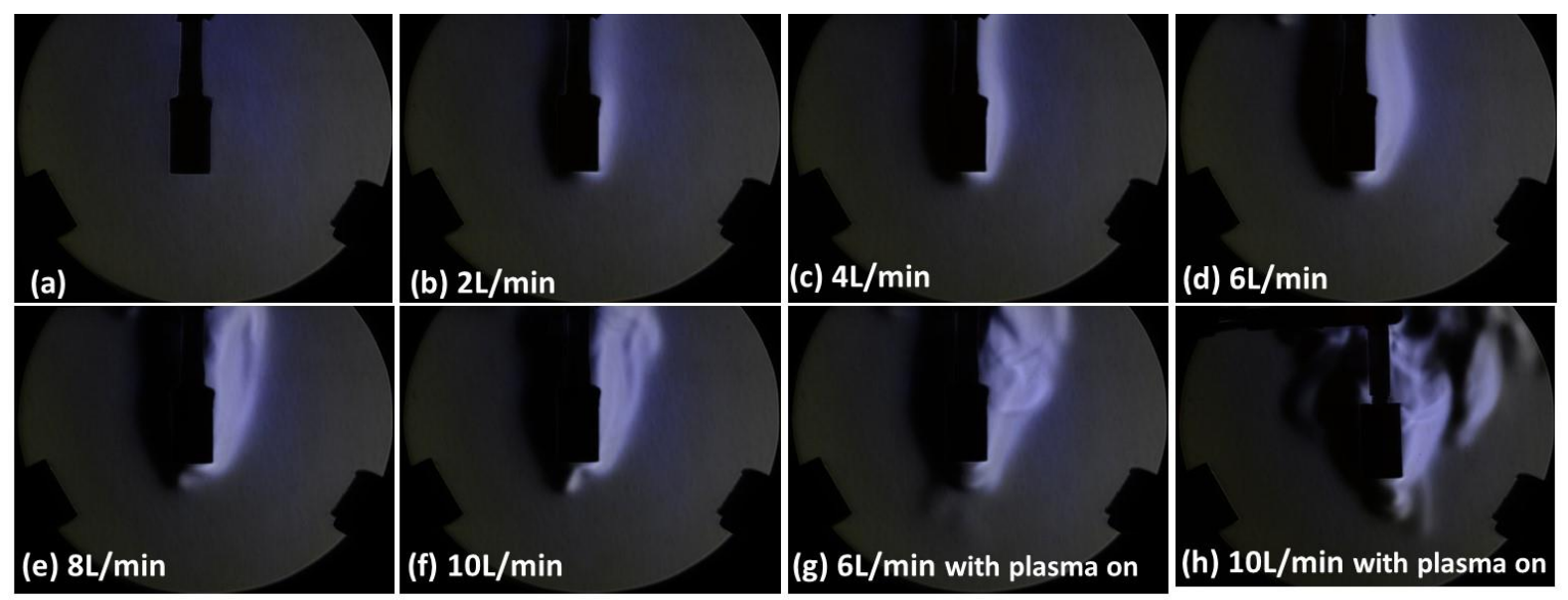

Figure 4. Schlieren images of helium flow into ambient air through the gas diffusor without/with plasma on. Gas flow rate is indicated in each image

Figure 5 shows the waveforms of applied voltage and plasma current with a fixed gas flow rate of $10.5 \mathrm{~L} / \mathrm{min}$, which correspond to the typical plasma modes in Figure 3. The plasma current comprises displacement current and conduction current. Both currents increase with 
the increasing applied voltage. Compared with the plasma ring mode, in the ray-like plume and swing-like plume modes, the plasma current shows a spike signature superimposing on the displacement current. In the transition from the ray-like plume to swing-like plume, the current spike broadens and its amplitude raises over 5mA as shown in Figure 5. By changing the applied voltage in each fixed gas flow rate and examining the discharge mode through current signature and plasma images, the parameter boundary (average applied voltage) between each mode is evaluated. No plasma plume means either no discharge or plasma ring mode. The mapping of parameter space in Figure 6 shows that the minimal gas flow rate and low applied voltage favors the generation of ray-like plume mode.


Figure 5. Applied voltage and current waveforms of the porous plasma jet of different discharge modes 


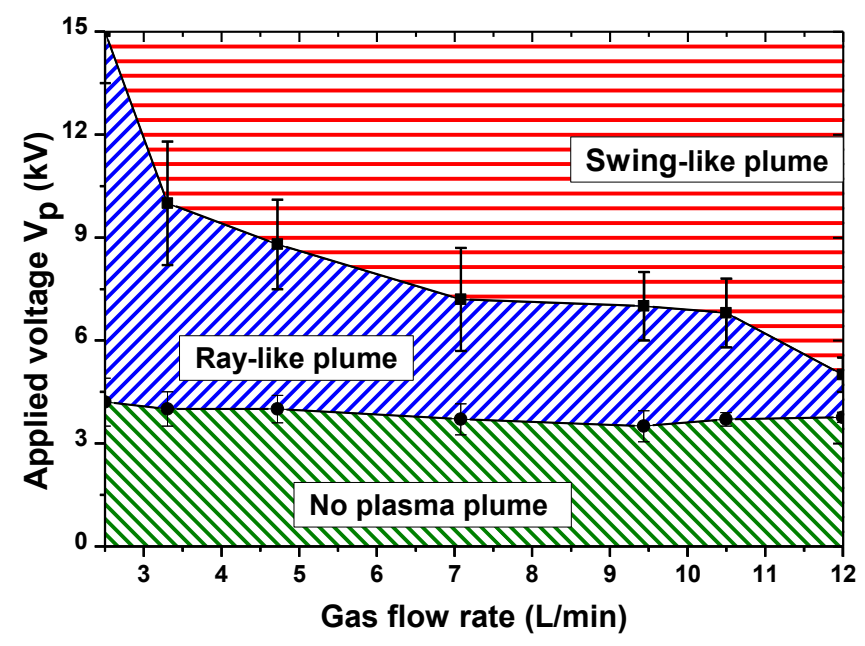

Figure 6. Mapping of discharge parameter region of the porous plasma jet for different discharge modes
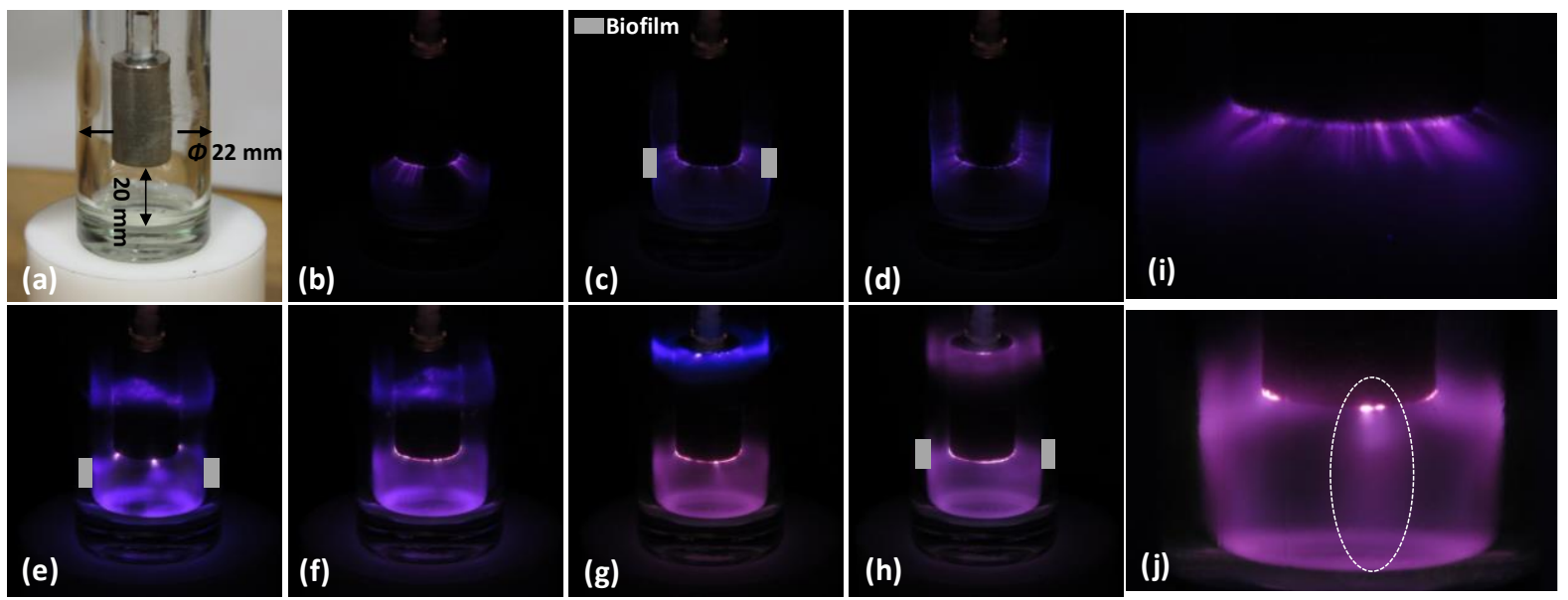

Figure 7. Photograph of gas diffusor inside a glass vial (a), porous plasma jet induced discharge at (b) $1.2 \mathrm{~L} / \mathrm{min}$, (c) $1.6 \mathrm{~L} / \mathrm{min}$, (d) $2 \mathrm{~L} / \mathrm{min}$, (e) $2.4 \mathrm{~L} / \mathrm{min}$, (f) $2.8 \mathrm{~L} / \mathrm{min}$, (g) $3.2 \mathrm{~L} / \mathrm{min}$, and (h) 4.7 L/min with the applied voltage of (Vp) $12.8 \mathrm{kV}$. (i) and (j) are close-up images showing filaments and glow-like region. Taken by Canon PowerShot SX60 HS, Exposure time 1/3s, ISO 200 The grey bar in (c), (e) and (h) indicates the position of biofilm adherent on the inner surface of the glass vial

One of the main aims of the proposed porous plasma jet is to inactivate an adherent biological contaminant on the inner surface of a tubular shape. To know the proper discharge parameter, initial studies focused on the discharge pattern of porous plasma jet induced discharge inside the glass vial (inner diameter $22 \mathrm{~mm}$ ). Figure 7 shows a series of discharge images under different gas flow rates in the case of fixed applied voltage $\left(V_{p}\right) 12.8 \mathrm{kV}$. In the case of low gas flow rate $(<2 \mathrm{~L} / \mathrm{min})$ radial discharge filaments eject from the gas diffusor and imping onto the glass vial surface, as shown in Figure 7(b)-(d). The discharge filaments as shown in 
Figure 7(i) mainly jet from the bottom edge of the gas diffusor due to the low gas flow rate and the electric field edge effect. With a slightly increased gas flow rate, the distribution region of the radial discharge filaments gradually expands upwards along the gas diffusor. Upon further increase of the gas flow rate (Figure 7(e)-(f)), the entire lower part of the glass vial under the gas diffusor is lighted by the discharge channel and two plasma regions are observed. The upper region consists of uniformly distributed radial plasma jets around the gas diffusor, which brush the glass vial inner surface. The seemingly uniform lower plasma region comprises multiple glow-like surface discharge regions along the glass vial surface as shown in Figure 7(j). In the case of higher gas flow rate $(>2.8 \mathrm{~L} / \mathrm{min})$, two different color plasma regions can be clearly seen as shown in Figure $7(\mathrm{~g})$. This is due to the change of gas composition from air to helium inside the glass vial. The lower plasma region is surface helium gas discharge; while the upper part is volume discharge in air which contains reactive nitrogen and oxygen species. When the gas flow rate is further increased both plasma regions are pink which exhibits helium dominated discharges (helium-filled discharge tube) as shown in Figure 7 (h). Overall, the porous plasma jet-induced discharge inside the glass cylinder has three typical modes, which are filament discharge mode (Figure 7 (b)- (d)), double region mode (Figure 7 (e)- (f))) and double region helium dominated mode (Figure 7(g)- (h)). These modes are named as mode 1,2 and 3, respectively.

The different discharge modes inside the glass vial may be tuned by varying the applied voltage. Discharge parameter region for porous plasma jet-induced discharge mode types inside the glass vial is mapped in Figure 8. In the case of high gas flow rate, mode 1 and 2 easily transit into mode 3. The apparent color difference from plasma emission suggests the different optical emission spectrum (OES) from different discharge modes. In the case of the low gas flow rate, the discharge filaments show the similar color as the air corona discharge. With the increasing gas flow rate, the plasma region color gradually changes to pink through the transition of violet. Figure 9(a) shows OES from different discharge modes which 
corresponds to Figure 7. In mode 2 and 3, the plasma emission was collected in the lower plasma region. Major identified spectral bands are from molecular nitrogen second positive systems $N_{2}(C-B)$ band, first negative systems $N_{2}^{+}(C-B)$ band, hydroxyl band of $O H(A-X)$, atomic helium (He I) and atomic oxygen $(O I)$. Figure 9(b) shows the change of OES intensities for selected bands of $N_{2}^{+}(C-B)$, He I and $O$ I with the gas flow rate. It is found that with the increasing helium flow rate the spectral intensities of $N_{2}^{+}(C-B)$ and $O I$ firstly increase and then decease with the increasing of $\mathrm{He} \mathrm{I}$ band intensity. The intensities of $N_{2}^{+}(C-B)$ and $O I$ bands reach maximum in the medium flow rate region (mode 2 discharge region). This is due to the increased air ionization region with the addition of helium gas compared with the smaller discharge region in mode 1 discharge.

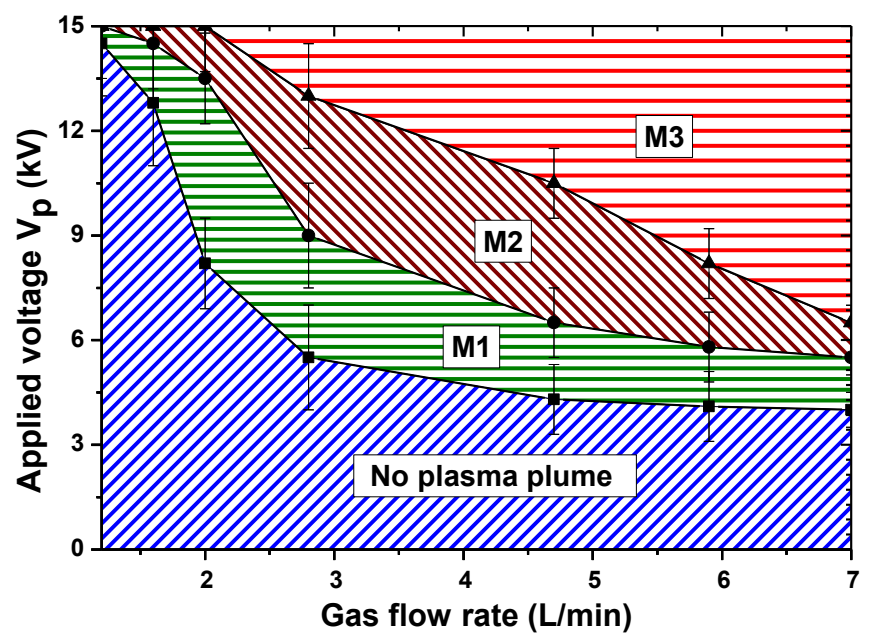

Figure 8. Mapping of discharge parameter region for porous plasma jet-induced different discharge modes inside the glass vial. 


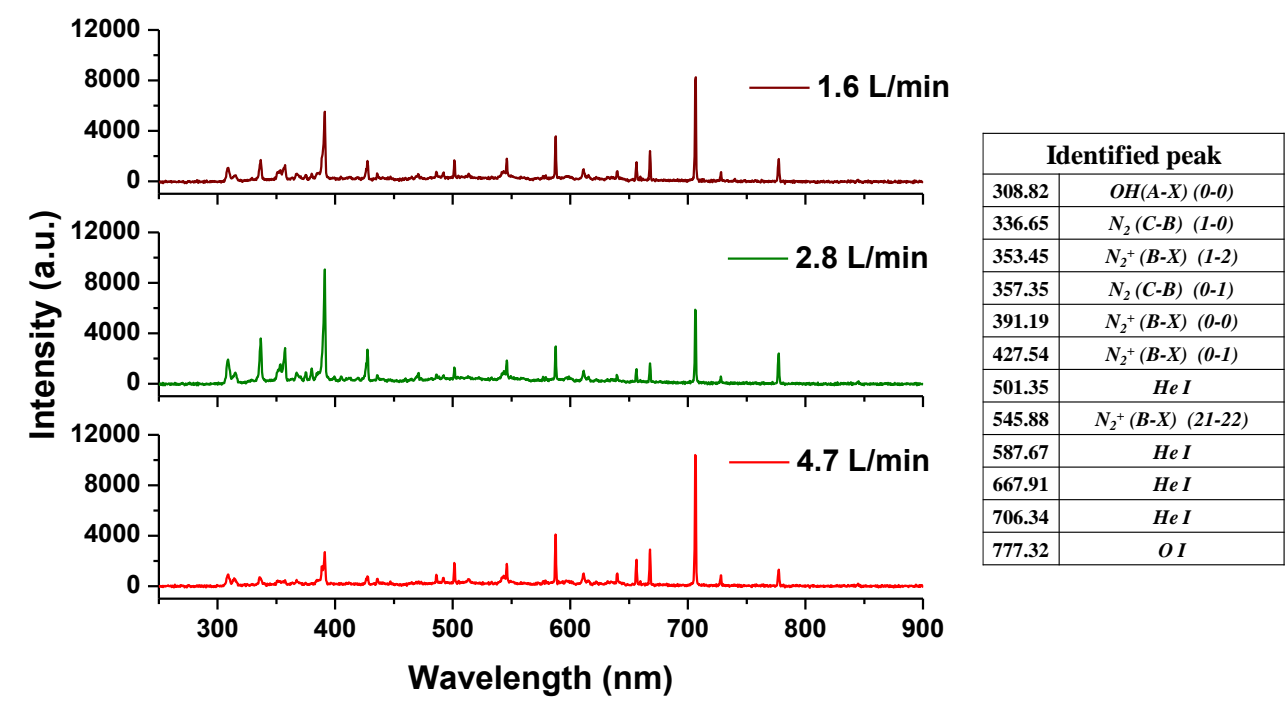

(a)

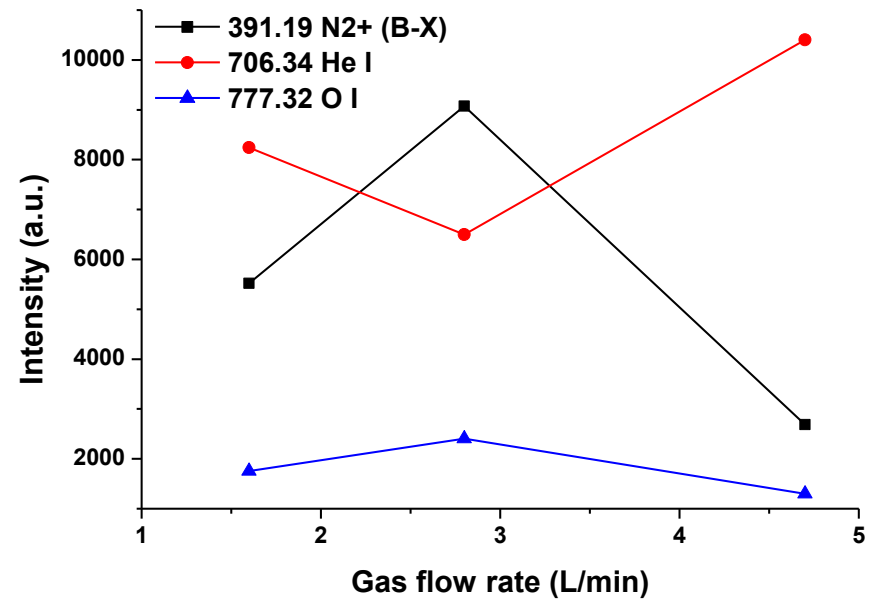

(b)

Figure 9. (a) OES of porous plasma jet-induced different discharge modes inside the glass vial, (b) change of OES intensities of $N_{2}^{+}(C-B)$, He I and $O$ I bands with the gas flow rate

\subsection{Biofilm inactivation}

The inactivation efficacy of 5 min of plasma treatments with three discharge modes was evaluated against $P$. aeruginosa 24 h biofilms formed on the inner surface of the glass vials. Control biofilms were exposed to corresponding helium gas flow treatment without discharge. In each mode the applied voltage $\left(V_{p}\right)$ was fixed at about $12.8 \mathrm{kV}$, the gas flow rate was set to 1.6 L/min, 2.8 L/min and 4.7 L/min as depicted in Figure 7 (c), (e) and (h), respectively. In 
each mode, the gas diffuser position was adjusted with respect to the biofilm position to ensure that the biofilm was treated in the discharge plasma region.

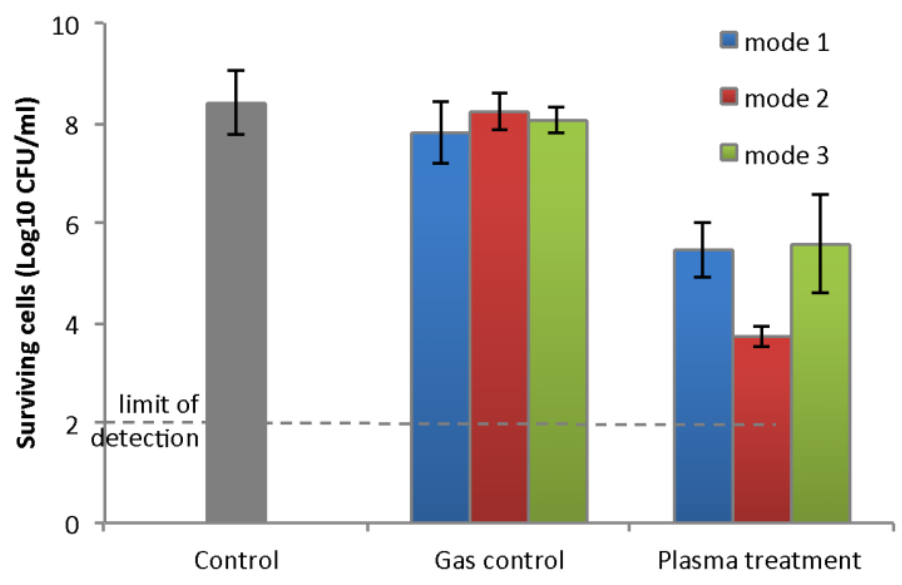

Figure 10. Surviving populations of $P$. aeruginosa $24 \mathrm{~h}$ biofilm after $5 \mathrm{~min}$ plasma treatments with discharge mode 1, 2 and 3, estimated by colony count assay



Figure 11. Surviving populations of $P$. aeruginosa $24 \mathrm{~h}$ biofilm after 5 min plasma treatments with discharge mode 2 , estimated by metabolic activity assay (XTT) and colony count assay.

Reductions of $P$. aeruginosa 24 h biofilms due to plasma treatment are presented in Figure 9. Initial bacterial cell populations attached on the inner surface of the vial after $24 \mathrm{~h}$ of incubation reached $8.4 \log _{10} \mathrm{CFU} / \mathrm{ml}(\mathrm{n}=13)$. These numbers decreased but insignificantly due to the action of helium gas flow without ignition of plasma. Discharge plasma treatments for 5 min with mode 1 and 3 considerably reduced bacterial numbers by 2.4 and $2.5 \log _{10}$ $\mathrm{CFU} / \mathrm{ml}$, respectively, by comparison to corresponding gas control. Plasma treatment with mode 2 was the most effective, reducing bacterial cells within the biofilm by $4.5 \log _{10}$ $\mathrm{CFU} / \mathrm{ml}$. This may be due to higher yield of reactive nitrogen and oxygen species, which are 
reflected from the higher spectral intensities of $N_{2}^{+}(C-B)$ and $O I$. In addition, ozone which is a prominent long-living reactive species generated by most atmospheric pressure plasma sources operated in ambient air might also play a role for the biofilm inactivation effect. The ozone concentration in effluents of plasma jets in ambient air has been mostly measured with helium (or Ar) and oxygen gas admixture ${ }^{[23-25]}$. It is reported that with the oxygen ratio up to $10 \%$, the volume-averaged ozone concentration is in the order of $10^{15} \mathrm{~cm}^{-3}$, which is from a few tens of ppm to a few hundreds of ppm. Compared with the oxygen admixture helium plasma jet, the porous plasma jet is operated with pure helium, consequently resulting in a lower ozone concentration. Atomic oxygen is a precursor for ozone formation. Therefore, it may be suggested that the mode 2 has the highest ozone yield due to its highest spectral intensity of $O I$. Excessive concentrations of RONS can be damaging to cells if present for too long and/or in too high a concentration ${ }^{[3]}$. Further, to confirm the viablility of cells within the biofilms, XTT assay was performed to study metabolic activity of P. aeruginosa following plasma exposure. As shown in Figure 10, the percentage of metabolic capacity of cells within biofilms considerably decreased by $40 \%$ after plasma treatment as compared to the corresponding gas control. XTT assay demonstrated slightly higher cell viability than estimated by the colony count assay, where culturability of cells was reduced by a half. It has been previously reported by our group and by other co-workers that plasma can result in bacterial cells to enter viable but non-culturable state $(\mathrm{VBNC})^{[26-28]}$, a survival state in which bacterial cells are no longer capable to form colonies on media but still able to perform respiration, gene transcription and protein synthesis ${ }^{[29]}$. The glass vial wall temperature was measured by an infrared thermometer (Fluke 561). The temperature was measured at different angles around the axis of the glass vial and averaged to the value. The highest wall temperature increased to about $47^{\circ} \mathrm{C}$ from the initial $22^{\circ} \mathrm{C}$ after mode 2 discharge treatment, while the wall temperature raised to about $40^{\circ} \mathrm{C}$ and $35^{\circ} \mathrm{C}$ after mode 3 and mode 1 plasma treatments, respectively. Additional experiments were conducted in order to assess the effect 
of temperature on viability of $P$. aeruginosa biofilms. On average $(\mathrm{n}=3) 9.0( \pm 0.1)$ and $8.6( \pm$

0.5) $\log _{10} \mathrm{CFU} / \mathrm{ml}$ were recovered for the controls and after heat treatment, respectively, with

\section{$0.4 \log _{10} \mathrm{CFU} / \mathrm{ml}$ reduction observed, confirming that this temperature was not sufficient to}

inactivate the cells. Similar effect of elevated temperatures on $P$. aeruginosa biofilms

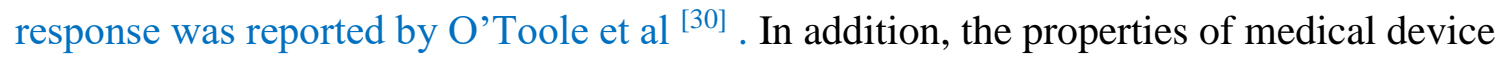

materials or industrial pipework or tubing are usually such that temperatures of up to $50{ }^{\circ} \mathrm{C}$ do not cause adverse effects.
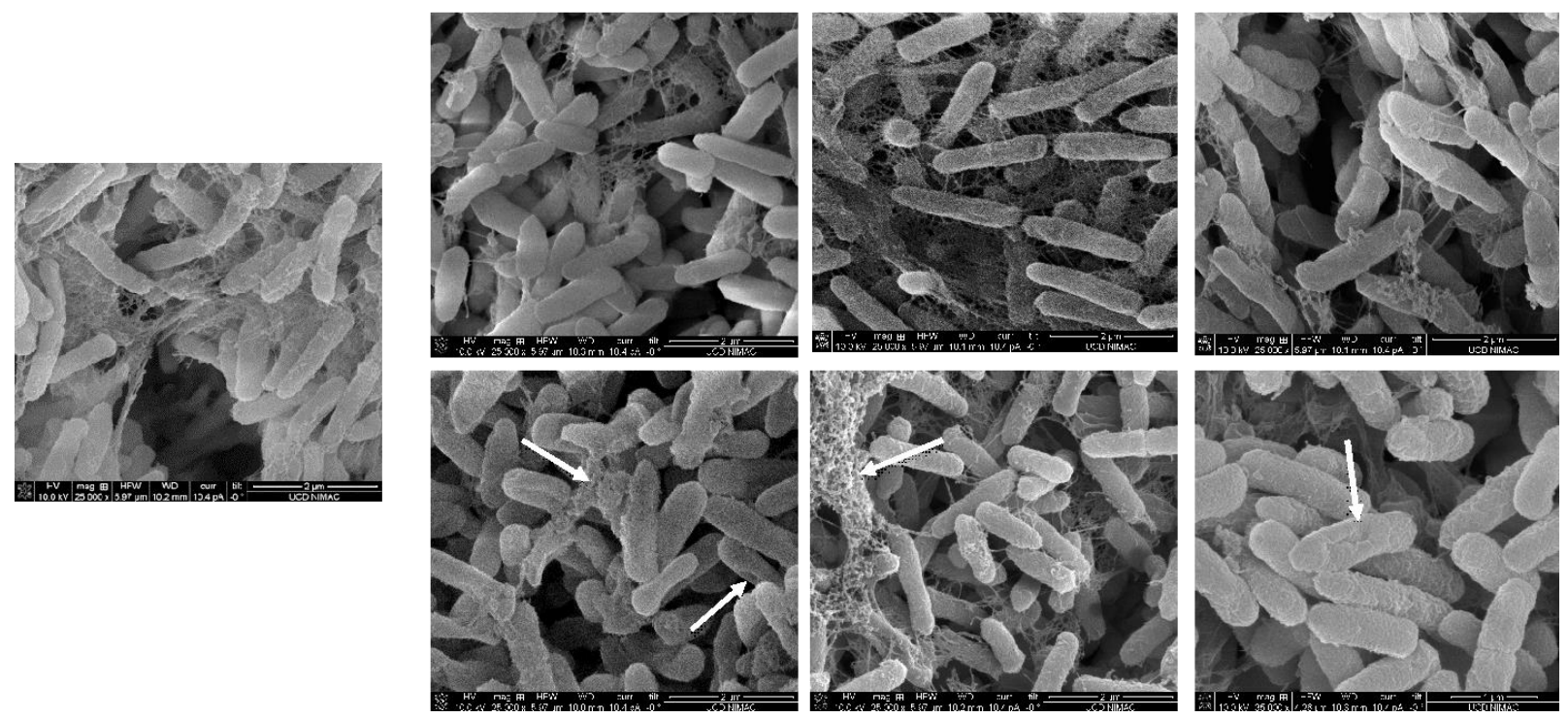

Figure 12. SEM images of $P$. aeruginosa $24 \mathrm{~h}$ biofilms: untreated control (left), control biofilms subjected to 5 min helium gas flow corresponding to mode 1,2 and 3 without plasma ignition (top panel) and biofilms treated with 5 min plasma discharge with mode 1, 2 and 3 (bottom panel). White arrows indicate biofilm matrix disintegration and holes on the surface of cells.

SEM analysis was performed to examine the effects of porous plasma jet treatment on $P$. aeruginosa biofilm morphology. The cells of the untreated control biofilm were intact and interconnected with each other by the biofilm matrix components (Figure 11, left image). The damage of bacterial cells, i.e., presence of holes on the wall of some of the bacterial cells after exposure to mode 1 and 3 and destruction of the biofilm matrix following exposure to mode 1 and 2 (white arrows) were observed (Figure 11, bottom panel). In comparison, complete disintegration of cells and biofilm matrix was earlier reported where plasma generated using 
high voltage dielectric barrier discharge system converted healthy P. aeruginosa biofilms into a 'sponge-like,' irregularly shaped structures ${ }^{[26]}$. In the present work, the difference between destructive effects on the biofilm matrix of the three discharge modes was insignificant, as the majority of cells visually remained intact. Similar findings were reported by Xu et al ${ }^{[17]}$ where complete disintegration or removal of Staphylococcus cells was not clearly observed by SEM. On one hand, in this circumstance, when high inactivation levels of biofilms was associated without obvious disintegration of cell and matrix components may prove useful, as part of a contamination control strategy, where confirmed inactivation is necessary prior to biofilm removal, to mitigate cross contamination or dislodgement of viable cells to a subsequent location. On the other hand, further research is required in order to elucidate inactivation pathways, to characterize matrix exo-polymers before and after plasma treatment and to describe biochemical damages to biofilm matrix components. Furthermore, in this work a small fraction of extracellular polymeric substances were still visible within inner layers of cells after the plasma treatment generated by mode 2 , which could indicate that plasma treatment had higher impacts on the outer layers of cells, leaving extracellular components as well as cells in the inner layers intact. Therefore, system optimization is still required to achieve efficient inner surface sterilization and decontamination for the specific biofilm risks within the medical device, food and pharmaceutical manufacturing sectors.

\section{Conclusion}

We present a helium porous plasma jet based on a cylindrical metal gas diffuser with the pore size of $10 \mu \mathrm{m}$. Porous plasma jets ignited in the ambient air and inside a glass vial have been investigated. By varying the applied voltage and gas flow rate, porous plasma jet can operate with three typical discharge patterns in the ambient air, namely localized plasma ring, laminar plume and turbulent plume. When it is placed inside a glass vial, the porous plasma jet induces three different discharge modes, which are filament discharge mode, double region mode and double region helium dominated mode. The inactivation effect of $P$. aeruginosa 
biofilm adherent on the inner surface of the glass vial by the porous plasma jet treatment was evaluated, showing that porous plasma jet induced air surface discharge significantly reduces bacterial cells within the biofilm by greater than $4 \log _{10} \mathrm{CFU} / \mathrm{ml}$ in comparison with cell reductions of 2.4 and $2.5 \log _{10} \mathrm{CFU} / \mathrm{ml}$ by plasma treatments with filament discharge mode and helium dominated discharge mode. The porous plasma jet presented could provide an efficient alternative for the inner surface sterilization and decontamination for relevant sectors, such as medical device, food and pharmaceutical manufacturing industries. Acknowledgements: This publication has emanated from research supported in part by a research grant from Science Foundation Ireland (SFI) under the Grant Number SFI/16/BBSRC/3391 and the BBSRC under the Grant Reference BB/P008496/1 and in part under SFI Grant Number 14/IA/2626.

Received: ((will be filled in by the editorial staff)); Revised: ((will be filled in by the editorial staff)); Published online: ((please add journal code and manuscript number, e.g., DOI: 10.1002/ppap.201100001))

Keywords: biofilm; discharge mode; gas diffusor; inner surface; porous plasma jet

[1] A. Fridman, G. Friedman, Plasma Medicine, Wiley, Hoboken, NJ, USA 2013.

[2] T. v. Woedtke, S. Reuter, K. Masur, K-D. Weltmann, Phys. Rep. 2013, 530, 291.

[3] X. Lu, G.V. Naidis, M. Laroussi, S. Reuter, D.B. Graves, K. Ostrikov, Phys. Rep. 2013, 530, 291.

[4] J. Ehlbeck, U. Schnabel, M. Polak, J. Winter, T. V. Woedtke, R. Brandenburg, T. V. Hagen, K.D. Weltmann, J. Phys. D: Appl. Phys. 2011, 44, 013002.

[5] G. Lloyd, G. Friedman, S. Jafri, G. Schultz, A. Fridman, K. Harding, Plasma Process. Polym. 2010, 7, 194. 
[6] M. G. Kong, G. Kroesen, G. Morfill, T. Nosenko, T. Shimizu, J. v. Dijk, J. L. Zimmermann, New J. Phys. 2009, 11, 115012.

[7] N. N. Misra, O. K. Schluter, P. J. Cullen, Cold Plasma in Food and Agriculture: Fundamentals and Applications, Academic Press, 2016.

[8] D. Yan, H. Cui, W. Zhu, N. Nourmohammadi, J. Milberg, L .G. Zhang, J. H. Sherman, M. Keidar, Scientific Reports 2017, 7, 4479.

[9] M. Ishaq, M. Evans, K. Ostrikov, Int. J. Cancer 2013, 134, 1517.

[10] S. Kitazaki, N. Hayashi, IEEE Trans. Plasma Sci. 2008, 36, 1304.

[11] S. Kitazaki, A. Tanaka, N. Hayashi, Vacuum 2014, 110, 217.

[12] M. Polak, J. Winter, U. Schnabel, J. Ehlbeck, K.D. Weltmann, Plasma Process. Polym. 2012, 9, 67.

[13] J. Winter, R. Brandenburg, K.D. Weltmann, Plasma Sources Sci. Technol. 2015, 24, 064001.

[14] J. W. Costerton, P. S. Stewart, E. P. Greenberg, Science. 1999, 264, 1318.

[15] L. H. Stoodley, J. W. Costerton, P. Stoodley, Nature Rev. Microbiol. 2004, 2, 95.

[16] R. Matthes, I. Koban, C. Bender, K. Masur, E. Kindel, K.D. Weltmann, T. Kocher, A. Kramer, N.O. Hubner, Plasma Process. Polym. 2013, 10, 161.

[17] Z. Xu, J. Shen, Z. Zhang, J. Ma, R. Ma, Y. Zhao, Q. Sun, S. Qian, H. Zhang, L. Dong, C. Cheng, P. K. Chu, W. Dong, Plasma Process. Polym. 2015, 12, 827.

[18] X. Pei, X. Lu, J. Liu, D. Liu, Y. Yang, K. Ostrikov, P.K. Chu, Y. Pan, J. Phys. D: Appl. Phys. 2012, 45, 165205.

[19] D. Ziuzina, D. Boehm, S. Patil, P. J. Cullen, Paula Bourke, PLoS One. 2015, 10, 0138209.

[20] M. Modic, N.P. McLeod, J.M. Sutton, J. L. Walsh, Int. J. Antimicrob. Agents. 2017, 49,375 .

[21] E. Peeters, H. J. Nelis, T. Coenye, J. Microbiol. Methods. 2008, 72, 157. 
[22] A. Los, D. Ziuzina, D. Boehm, P. J. Cullen, P. Bourke, Innov Food Sci Emerg

Technol. 2017, 44, 36-45.

[23] J. Winter, M. Dunnbier, A. S. Bleker, A. Meshchanov, S. Reuter, K. D. Weltmann, J. Phys. D: Appl. Phys. 2012, 45, 385201

[24] B. L. Sands, B. N. Ganguly, J. Appl. Phys. 2013, 114, 243301.

[25] S. Reuter, J. Winter, S. Iseni1, S. Peters, A. S. Bleker, M. Dunnbier, J. Schafer, R.

Foest, K. D. Weltmann, Plasma Sources Sci. Technol.. 2012, 21, 034015.

[26] D. Ziuzina, S. Patil, P.J. Cullen, D. Boehm, P. Bourkea, Plasma Medicine 2014, 4, 137.

[27] M. Y. Alkawareek, Q. Th. Algwari, G. Laverty, S. P. Gorman, W. G. Graham, D. O. Connell, B. F. Gilmore, PLoS One 2012, 7, e44289.

[28] G. B. Marino, J. Appl. Microbiol.. 2012, 112, 412.

[29] D. Pinto, M. A. Santos, L. Chambe, Crit Rev Microbiol. 2015, 41,61.

[30] A. O. Toole, E. B. Ricker, E. Nuxoll, Biofouling 2015, 31, 665. 


\section{Graphical Abstract}

The table of contents entry should be fifty to sixty words long (max. 400 characters), and the first phrase should be bold. The entry should be written in the present tense and impersonal style. The text should be different from the abstract text.

We present a helium porous plasma jet. With the proper discharge mode, it significantly inactivates $P$. aeruginosa biofilm adherent to the inner surface of a glass vial by greater than 4 $\log _{10} \mathrm{CFU} / \mathrm{ml}$. The porous plasma jet presented could provide an efficient alternative for the inner surface sterilization in relevant sectors, such as medical device, food and pharmacy industries.

Peng Lu, Dana Ziuzina, PJ Cullen, Paula Bourke*.

Title: Inner Surface Biofilm Inactivation by Atmospheric Pressure Helium Porous Plasma Jet

\section{ToC Figure}

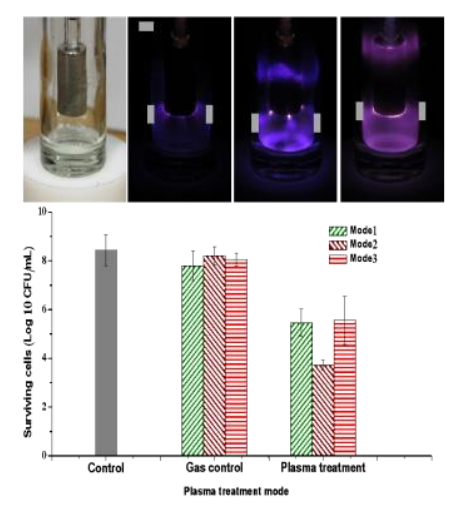

